\section{Viele Geburtshelfer leiden an PTSD}

Sterbende oder schwer geschädigte Neugeborene, tote oder schwer verletzte Mütter - in einer Befragung von 700 ärztlichen Geburtshelfern und 1400 Hebammen antworteten $84 \%$ der Ärzte und $71 \%$ der Hebammen, so etwas schon erlebt zu haben. $43 \%$ von ihnen berichteten, während der Ereignisse von Furcht, Hilflosigkeit oder Panik ergriffen worden zu sein. In beiden Berufsgruppen betrug der Anteil von Personen, die Symptome einer partiellen posttraumatischen Belastungsstörung (PTSD) zeigten, 15\%. 7\% der Ärzte sowie $5 \%$ der Hebammen erfüllten die Symptomkriterien einer voll ausgeprägten PTSD.

BJOG 2016, online 24. August

\section{Schlafapnoe bringt vom Weg ab}

129 unbehandelte Patienten mit obstruktivem Schlafapnoe-Syndrom (OSAS) absolvierten jeweils zwei Autofahrten und 79 gesunde Kontrollpersonen jeweils eine Fahrt mit dem Simulator. Dabei wurde beurteilt, wie gut die Probanden die Spur hielten. Obwohl die Teilnehmer beider Gruppen im Mittel schon seit über 30 Jahren den Führerschein besaßen, hatten OSAS-Patienten mehr Schwierigkeiten; die Standardabweichung von der Fahrspur war signifikant größer als in der Kontrollgruppe. Dementsprechend fielen $20 \%$ beim Fahrtest durch, in der Vergleichsgruppe kein Teilnehmer.

ERS, London, 3.-7. September 2016

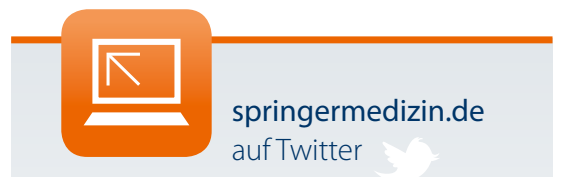

Auch im Herbst heißt es wieder:

Lauschen Sie unserem Zwitschern und werden Sie zum Follower!

twitter.com/springermedizin

Frauen stärker betroffen

\title{
Infarkt verdirbt vielen die Lust
}

Nach einem Herzinfarkt will es selbst bei vielen jüngeren Patienten im Bett nicht mehr so recht klappen. Wie eine Studie zeigt, könnten Gespräche mit dem behandelnden Arzt die Situation womöglich verbessern.

Im Zuge einer Längsschnittstudie hat sich ein Wissenschaftlerteam mit dem Problem befasst, wie sich ein Herzinfarkt auf das Sexleben jüngerer Patientinnen und Patienten auswirkt. 1889 Frauen und 913 Männer aus den USA und Spanien im Alter zwischen 18 und 55 Jahren (Median: 49 Jahre) nahmen an der Untersuchung teil. Von den vorher aktiven Männern hatten $64 \%$ einen Monat nach dem Infarkt wieder Sex, nach einem Jahr waren es 94\%. Bei Frauen lagen die Raten durchweg niedriger, nämlich bei $55 \%$ nach einem Monat und $91 \%$ nach einem Jahr. 60\% der Frauen, die vor und nach dem Infarkt nicht auf Sex verzichteten, klagten dennoch über sexuelle Probleme. Selbst $42 \%$ derjenigen, die zuvor keine Probleme gehabt hatten, entwickelten im ersten Jahr nach dem Myokardinfarkt Schwierigkeiten.

Als gewisser Schutz erwies es sich, früh mit einem Arzt über das Sexualleben zu sprechen. Wer dies nicht schon im Lauf des ersten Monats nach dem Infarkt tat, hatte ein um 51\% erhöhtes Risiko, erst spät wieder auf den sexuellen Geschmack zu kommen.

JAMA Cardiol 2016, online 31. August;

doi: 10.1001/jamacardio.2016.2362

\section{Diagnose meist korrekt}

\section{Kleine Nierentumoren bioptisch beurteilen}

Bei Patienten mit Nierentumoren unter $4 \mathrm{~cm}$ Durchmesser sind Biopsien vor einer möglichen Operation zur Vermeidung unnötiger Eingriffe durchaus sicher durchführbar. Sie erlauben in den meisten Fällen eine diagnostische Aussage, wie die Ergebnisse einer retrospektiven Studie bestätigen.

Mithilfe der modernen Bildgebung des Abdomens gelingt es immer häufiger, auch kleine Nierenzellkarzinome $(<4 \mathrm{~cm})$ zu entdecken. Für die Studie wurden die Befunde von 351 Patienten mit Nierenzellkarzinom ausgewertet, die zwischen 2011 und 2015 biopsiert worden waren, um die zuvor entdeckten kleinen Tumoren zu charakterisieren. Nach Ausschluss weiterer Befunde wurden schließlich die
Ergebnisse von insgesamt 388 Biopsien, die von 373 Nierentumoren stammten, für die Analyse verwendet. Primärer Endpunkt der Studie waren die Raten an Biopsien, die zu Diagnosen bzw. nicht zu einer Diagnose führten, und die Ermittlung von Faktoren, die eine Diagnose zum Zeitpunkt der Erstbiopsie ermöglichen.

Nach Angaben der Urologen konnte durch Kombination der Ergebnisse eine Diagnoserate von $91 \%$ erreicht werden. Die häufigste maligne Diagnose war ein klarzelliges Nierenzellkarzinom, am zweithäufigsten ein papilläres Nierenzellkarzinom. In keinem Fall kam es nach Angaben der Ärzte aufgrund der Feinnadelbiopsien zur Streuung von Krebszellen.

(rb)

BJU Int 2016; online 7. September; doi: 10.1111/bju.13630 